\title{
Preface
}

\section{Rhinoplasty: Education Through Multispecialty Collaboration}

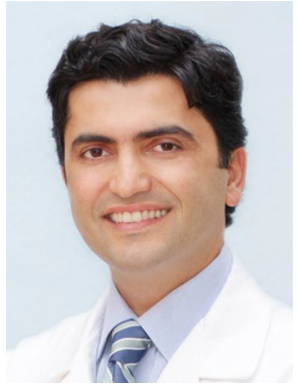

Babak Azizzadeh, MD, FACS Editor

It is a great honor to be part of this special issue of Clinics in Plastic Surgery dedicated to multispecialty rhinoplasty. I embarked several years ago in partnership with Clinics in Plastic Surgery to develop publications that bring together viewpoints of different core specialists in order to provide the most up-to-date and advanced educational forum. The first two issues focused on eyelid, brow, and midface rejuvenation. This issue is solely dedicated to rhinoplasty and nasal surgery. Rhinoplasty has long been considered one of the most challenging operations in esthetic and reconstructive surgery. Although plastic surgeons and facial plastic surgeons have for decades worked alongside one another in teaching and practicing this beautiful surgery, very few publications have put forth techniques by experts in both specialties. This unfortunate fact has truly affected multispecialty education and collaboration. We hope that these series of journals will be the beginning of many more collaborative efforts by experts from various different specialties.

The guest editors, Drs Daniel Becker and Ronald Gruber, are among the most prolific rhinoplasty surgeons and educators in their respective fields of facial plastic surgery and plastic surgery.
They have done a remarkable job of putting together a rhinoplasty compendium that will serve both the novice and the experienced surgeon alike. I owe them a debt of gratitude that is beyond words. This project could not have been completed without the tireless effort of the editors at Elsevier, specifically Donald Mumford, Jessica McCool, and Mahalakshmi Narayanan. Finally, I would like to thank my wonderful family for supporting not just this effort but countless other projects. Their selfless sacrifice will never be forgotten.

Babak Azizzadeh, MD, FACS Center for Advanced Facial Plastic Surgery 9401 Wilshire Boulevard, Suite 650 Beverly Hills, CA 90212, USA

Department of Head and Neck Surgery David Geffen School of Medicine at UCLA Los Angeles, CA, USA

E-mail address: drazizzadeh@gmail.com Website: http://www.facialplastics.info (B. Azizzadeh) 